Pour citer ce document :

THIBAUD, Jean-Paul. Mouvement et perception des ambiances souterraines. Les annales de la recherche urbaine, juin 1996, $n^{\circ} 71$, pp. 144-152.

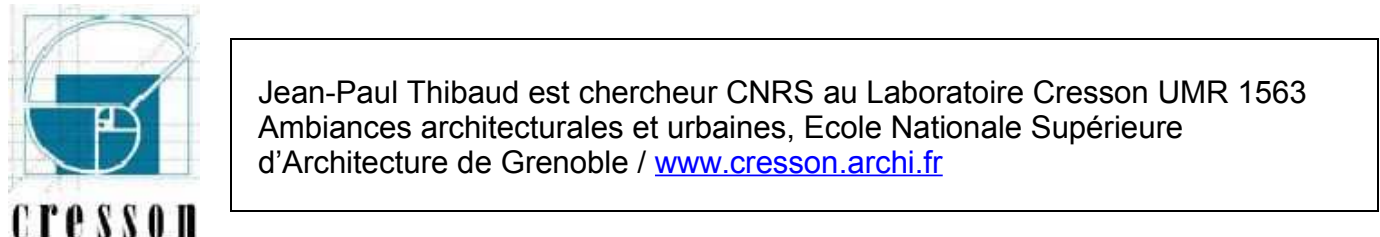

\title{
Mouvement et perception des ambiances souterraines
}

\section{Jean-Paul Thibaud}

La mobilité ne constitue pas seulement un des enjeux majeurs des espaces publics urbains, elle se donne comme un des critères essentiels conférant à l'espace urbain son caractère public. C'est ainsi que le lieu-mouvement peut être considéré comme l'idéal-type de l'espace public contemporain. Réduit parfois à un complexe d'interconnexion de différents modes de transport, le lieu-mouvement relève plus fondamentalement d'un espace de ressources et d'épreuves mobilisant l'activité perceptive et motrice des passants. A cet égard, on peut s'intéresser à l'"informationvoyageur" en mettant à l'épreuve les conditions de production, diffusion et réception des informations mises à disposition des voyageurs (Gare du Nord : Mode d'emploi, 1994). Il s'agit de tester la manière dont un site favorise le transit en délivrant des messages pertinents et en proposant des équipements adéquats. L'analyse du dispositif informationnel - annonce sonore, télépancartage, signalétique, cellule d'information, etc. - révèle alors le lien étroit qui se tisse entre l'espace de circulation et l'espace de communication.

Une autre approche permettant de saisir l'efficace locomoteur d'un site consiste à questionner ce dernier à partir de ses ambiances. D'une perspective en terme d'accès à l'information nous passons à une démarche accordant une place centrale aux schèmes sensori-moteurs qui s'actualisent dans le déplacement. De ce point de vue, l'espace urbain est appréhendé comme un milieu sensible qui donne prise à des orientations perceptives et des comportements moteurs spécifiques.

Affirmer que l'ambiance d'un site engage la motricité de ses usagers conduit à reformuler la question des lieux-mouvement. Plutôt que de partir d'une typologie de lieux dont la fonction principale est a priori de favoriser le transit des voyageurs (gares, stations de métro, complexes d'échanges), cette approche cherche à déceler ce qui dans l'environnement construit participe du mouvement des passants. Le propos n'est pas d'évaluer le bon fonctionnement d'un lieu-mouvement - au sens d'un lieu conçu prioritairement pour le déplacement -, il vise plutôt à comprendre quelles sont les composantes de l'espace urbain qui engagent l'activité motrice des citadins. Formulé autrement, il s'agit de rendre compte de l'efficace sensori-moteur d'un site donné, d'expliciter les offres et emprises d'un lieu en terme de déplacement, bref, à mettre en évidence les facteurs d'ambiance qui font d'un lieu un lieu-mouvement. 
Pour citer ce document :

THIBAUD, Jean-Paul. Mouvement et perception des ambiances souterraines. Les annales de la recherche urbaine, juin 1996, $n^{\circ} 71$, pp. 144-152.

Selon cette perspective, un lieu-mouvement n'est pas pré-donné et défini à partir de sa fonction de trafic, il repose sur des formes sensibles qui le constituent comme tel.

\section{Configurations sensibles en mouvement}

Sans doute est-ce à partir de la notion de configuration sensible que l'on peut faire valoir l'intentionnalité motrice engagée dans toute perception. Une configuration sensible peut être définie comme le surgissement d'une forme découlant de l'interaction entre un monde sensible, un langage naturel et une activité configurante. De ce point de vue, une configuration n'est pas réductible à ses composantes physiques, à ses propriétés narratives ou même aux actes perceptifs qu'elle suscite. Les qualités sensibles d'une ambiance n'acquièrent de sens qu'à partir du moment où elles mobilisent des styles de mouvement et se schématisent selon un contexte de description. Formulé autrement, la mise en forme sonore et lumineuse d'un site convoque simultanément le pas du passant, sa parole, ainsi que son oeil et son oreille. La notion de configuration sensible rassemble trois problèmes : le substrat matériel de l'environnement sensible, le rapport entre perception et motricité, la constitution langagière de la réalité.

- L'environnement sensible comme potentialité active. L'environnement sensible repose sur des dispositifs matériels qui mettent en jeu l'interaction entre le cadre bâti (formes architecturées, volumes, matériaux) et des signaux physiques (lumineux, acoustiques,...). La distribution de la lumière et du son n'est donc pas homogène dans l'espace, elle varie selon la morphologie des lieux. Si la structuration sensible du milieu urbain est toujours située et circonstanciée, toujours relative aux traits spécifiques du contexte local, elle occasionne aussi des manières d'être et d'agir spécifiques. Que l'on insiste sur la "prégnance climatique" d'un lieu (Augoyard, 1979), sur les "offrandes" de l'environnement lumineux (Gibson, 1986) ou sur la "puissance formante" de l'espace public (Quéré, 1995), tout concourt à penser l'environnement sensible en terme de potentialité active. Les qualités sensibles d'un site opèrent à la fois comme élément structurant de la perception et invitation à agir. Elles doivent être pensées comme des disponibilités en devenir. La perception du citadin n'est donc pas assimilable au regard qu'il porte sur les choses, elle est plus fondamentalement exploration active du monde sous la main, appropriation des ressources que lui offre le lieu.

- Le paraître moteur des phénomènes sensibles. Toute perception implique un "bougé", aussi infime soit-il, qui rend possible l'acte même de percevoir. En procédant par schématisation et en faisant appel aux ressources du corps, l'activité configurante mobilise conjointement et indissociablement des conduites perceptives et des performances motrices. A cet égard, la phénoménologie n'a de cesse de montrer l'unité fondatrice du "sentir" et du "se mouvoir" (Straus, 1989), le chiasme originaire de la perception et du mouvement (Merleau-Ponty, 1964). Plutôt que de partir de la dualité de l'objet et du sujet, de l'action et de la perception, il s'agit de mettre à jour leur co-appartenance en faisant admettre quelque chose comme un 
Pour citer ce document :

THIBAUD, Jean-Paul. Mouvement et perception des ambiances souterraines. Les annales de la recherche urbaine, juin 1996, $\mathrm{n}^{\circ} 71$, pp. 144-152.

paraître moteur. De ce point de vue, le mouvement n'est pas assimilable à un simple changement de lieu ou au déplacement dans l'étendue géométrique, il s'appuie sur l'immédiateté des qualités sensibles en même temps qu'il les révèle : "il revient donc au même de dire que la phénoménalisation procède du monde dans lequel le sujet est engagé par ses mouvements, ou que c'est le sujet moteur qui, en se portant vers le monde, le fait paraître" (Barbaras, 1992). La mise en mouvement du corps est à la fois investissement pratique du monde et "sensibilisation" de celui-ci.

- L'appartenance langagière de l'expérience sensible. Le langage n'est pas seulement un instrument permettant de rendre compte après coup d'une expérience vécue, de la représenter ou de la transmettre, il participe pleinement et immédiatement de celle-ci. Toute expérience est constituée conceptuellement et n'a de véritable existence que par et dans le langage qu'elle met en jeu. Les événements et les choses se manifestent sous un contexte de description, ils s'individuent sur la base de schèmes conceptuels variables selon les époques et les cultures. Sans doute revient-il à Hans-Georg Gadamer (1976) d'avoir montré l'entrelacs du langage et du monde : "Non seulement le monde n'est monde que dans la mesure où il s'exprime en une langue, mais la langue, elle, n'a sa véritable existence que dans le fait que le monde se donne présence en elle". Cette perspective est lourde de conséquence d'un point de vue méthodologique. Les comptes rendus de perception auxquels nous avons procédé ne nous donnent pas seulement accès aux qualités sensibles du site, ils expriment les processus de schématisation en oeuvre dans la perception et révèlent les opérations de configuration auxquels se prêtent les ambiances du lieu.

Compte tenu de ces remarques préliminaires, comment le pas du passant incorpore-t-il les qualités lumineuses ou sonore du site ? Comment celles-ci modulent-elles des manières de bouger et donnent-elles prise à des gestes spécifiques ? Peut-on arriver à typifier quelques configurations qui rendent compte de l'efficace sensori-moteur des ambiances souterraines?

\section{L'heuristique du Grand Louvre}

L'espace du Grand Louvre à Paris présente un intérêt heuristique particulier pour mettre en évidence l'offre motrice des ambiances souterraines. Haut-lieu de tourisme, ce site prestigieux propose un cadre de visite original qui manifeste un souci évident accordé aux qualités sensibles des lieux. L'extrême stylisation des formes architecturales, la grande cohérence dans l'utilisation des matériaux et le minimalisme recherché de l'aménagement produisent des unités d'ambiance simples et distinctives qui en facilitent l'analyse. Mais l'intérêt de ce terrain réside aussi dans son caractère paradoxal. A cet égard, trois caractéristiques principales méritent d'être relevées : l'ambiguité fondamentale de sa dimension souterraine, la disjonction entre les ambiances sonores et lumineuses, l'injonction paradoxale de circuler et de séjourner. 
Pour citer ce document :

THIBAUD, Jean-Paul. Mouvement et perception des ambiances souterraines. Les annales de la recherche urbaine, juin 1996, $\mathrm{n}^{\circ} 71$, pp. 144-152.

Premièrement, le Grand Louvre possède les traits remarquables d'un milieu souterrain : combinaison de clôture et de verticalité, environnement "artificiel", absence de façade et d'horizon, cadre bâti inextensible. Toutefois, le filtrage de la lumière naturelle dans certaines parties du site (Hall Napoléon, Place de la pyramide inversée) favorise l'interpénétration entre le sous-sol et la surface, remet en cause la délimitation physique entre l'intérieur et l'extérieur et introduit une ambiguïté quant au caractère souterrain de l'environnement lumineux ${ }^{2}$. Comme le disent eux-mêmes les visiteurs : "on ne sait plus si on est en surface ou bien vraiment en-dessous". Le pas du passant n'engage pas seulement le contact immédiat avec le sol, il se module aussi en fonction des caractéristiques de l'enveloppe souterraine et du traitement particulier de la verticalité.

Deuxièmement, ce milieu souterrain possède des propriétés différentes selon que l'on s'intéresse à sa composante sonore ou lumineuse. A titre anecdotique, commentant l'ambiance sonore du Fossé Charles $V$ en référence à ce qu'il voit, un visiteur trouve une formule très parlante : "c'est curieux, on dirait qu'il y a une erreur sur la bande-son". Outre la prédominance accordée à ce qui est donné à voir, le Grand Louvre propose une composition et un agencement des seuils selon deux modes bien distincts. Au niveau visuel, la perméabilité de l'enveloppe à la lumière naturelle favorise l'émergence de forts contrastes, suscite un marquage des lieux suivant leur degré de confinement et engage des variations significatives dans le temps (jour/nuit, temps ensoleillé/couvert). Par contre, l'environnement sonore fonctionne comme un microcosme coupé de l'extérieur, exacerbe les délimitations aux entrées et sorties, instrumente davantage des transitions progressives à l'intérieur du sous-sol. Cette disjonction de l'audible et du visible nous amène à considérer l'offre motrice des ambiances lumineuses ${ }^{3}$.

Troisièmement, tout se passe comme si le Grand Louvre fonctionnait à la fois comme lieu de transit et appareil de capture. A très forte fréquentation touristique, ce lieu vise à attirer nombre de visiteurs, à provoquer une affluence massive tout en évitant les risques d'encombrement auxquels il se prête. Ce paradoxe du séjour et de la mobilité se manifeste de multiples façons, tant au niveau sensible qu'au niveau des stratégies réglementaires, de l'information-voyageur ou des conditions matérielles de réception du public. D'un côté, on s'arrange pour favoriser la mobilité des visiteurs (interdiction de s'asseoir par terre, optimisation de la vitesse des transactions agents-usagers, quasi-absence de bancs, déplacement du contrôle des billets d'accès

1 En l'absence de terme approprié, Lewis Mumford (1934) inventa l'expression "manufactured environnement" pour désigner le caractère "artificiel" de l'environnement souterrain.

2 Cette utilisation de la lumière naturelle n'est pas sans rappeler l'ambiguïté des passages parisiens tels qu'ils sont décrits par Walter Benjamin (1989).

3 Dans l'idéal, il faudrait pouvoir comprendre comment le pas du passant parvient à résoudre et intégrer les discordances entre l'emprise sonore et l'emprise lumineuse du site. Toutefois, dans l'état actuel des connaissances et en l'absence d'une théorie transsensorielle cette exigence est sans doute prématurée. 
Pour citer ce document :

THIBAUD, Jean-Paul. Mouvement et perception des ambiances souterraines. Les annales de la recherche urbaine, juin 1996, $\mathrm{n}^{\circ} 71$, pp. 144-152.

au musée pour désengorger le hall d'accueil, etc.) ; de l'autre, on rend ceux-ci captifs du lieu (interception temporaire des passants à certains endroits, défaillance de la signalétique relative aux sorties, manifestations culturelles occasionnelles qui provoquent des engorgements, etc.). Par exemple, la place de la pyramide inversée se prête à de nombreux commentaires de ce type : "c'est un endroit où on pourrait rester, parce que c'est un bel endroit, mais on ne peut pas s'asseoir". Cette double contrainte constitue un élément central de la structuration sensible du site. Soumis à des impératifs opposés, cet espace exacerbe le caractère problématique de la mobilité en sous-sol.

\section{L'appréhension des surfaces lumineuses}

Le rapport de l'oeil et du pas met en jeu des dispositifs architecturaux plus ou moins complexes qui peuvent mobiliser des conduites opto-motrices de nature très différente. Toutefois, deux traits principaux s'appliquent à l'ensemble du site. D'une part, la qualité des matériaux - leur aspect lisse et glissant - favorise de toute évidence le déplacement : "l'utilisation du marbre est très intéressante parce qu'on glisse dessus", "cette notion de 'poli' on sent qu'on est encouragé à marcher", "j'insiste sur la matière, sur le côté lisse, aussi bien sur le sol que sur toutes les façades". D'autre part, la densité du public peut altérer les conditions de visibilité des passants et remettre en cause la fluidité de l'espace construit : "il y a très peu de gens qui vont dans notre sens, donc en fait on se heurte aux gens", "le passage vers le Louvre est très encombré, les gens se bousculent, c'est pénible pour moi", "ça stagne ici, les gens ne bougent pas, ils sont immobiles parce qu'ils font la queue". Cette interaction entre les qualités sensibles du site et le degré de fréquentation du public (photo 1) constitue le contexte commun aux quelques cas de figure décrits cidessous. 
Pour citer ce document :

THIBAUD, Jean-Paul. Mouvement et perception des ambiances souterraines. Les annales de la recherche urbaine, juin 1996, $n^{\circ} 71$, pp. 144-152.

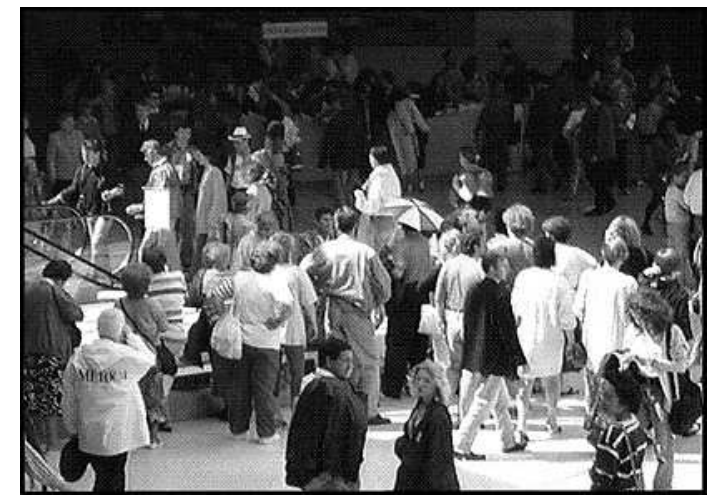

1. Hall Napoléon : la densité du public limite la portée du regard, diminue la visibilité du sol et restreint la mobilité.

InJONCTION AU DÉPLACEMENT : LE GLISSEMENT DU REGARD. Les couloirs aveugles du métro reliés au Grand Louvre fonctionnent pratiquement comme une injonction au déplacement : forte directionalité de l'espace produite par l'étroitesse du passage et la ligne de lumière au plafond, absence totale d'objets ou d'inscriptions retenant le regard, sol dépourvu de tout obstacle, surfaces opaques délimitant un champ de vision très restreint (photo 2).

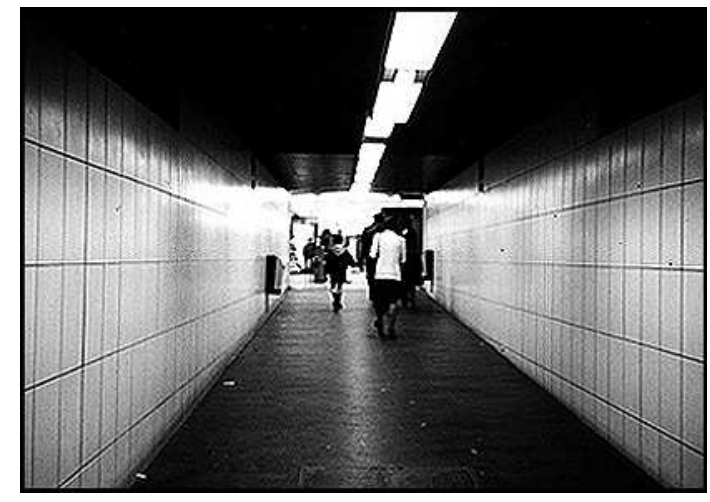

2. Couloir aveugle du métro : l'injonction au déplacement

La concision des descriptions effectuées par les passants et la stricte énumération des surfaces-couleurs témoigne de l'extrême dénuement du passage. Ici, point n'est besoin de s'arrêter, ou même de ralentir ; tout peut être vu d'un seul coup d'oeil et dit en une phrase : "couloir bien blanc, carrelage blanc, sol noir-gris et plafond bleu", "un couloir de métro, blanc au mur et bleu au plafond", "le plafond est bleu, sur les 
Pour citer ce document :

THIBAUD, Jean-Paul. Mouvement et perception des ambiances souterraines. Les annales de la recherche urbaine, juin 1996, $\mathrm{n}^{\circ} 71$, pp. 144-152.

côtés c'est tout blanc et des lumières blanches, toutes droites, qui indiquent la sortie". La parole sur l'espace glisse comme le regard sur les surfaces colorées, le regard ne peut s'échapper qu'en se centrant sur l'issue de sortie, le pas du passant ne fait que répéter ce que l'oeil a déjà accompli.

Ces effets de centration, glissement et anticipation du regard deviennent d'autant plus manifestes quand on compare les couloirs du métro à l'Allée de France. Bordée de vitrines, cette galerie souterraine - elle aussi cadrée - tend au contraire à dilater l'espace visuel, à solliciter la vision latérale et à offrir des occasions de pause (photo $3)$.

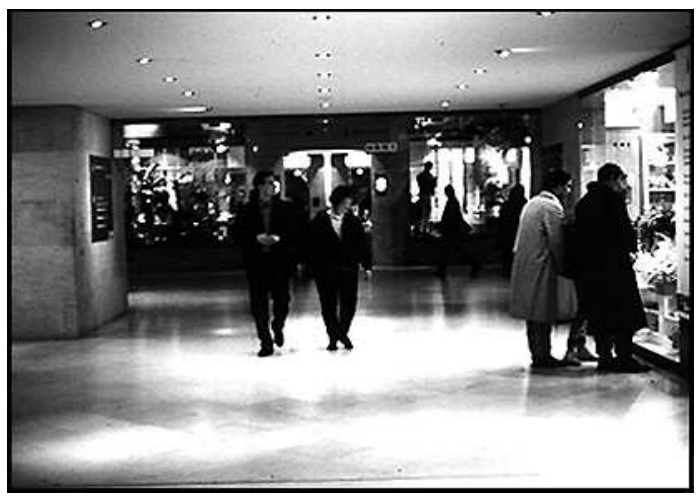

3. Allée de France : l'incidence de l'illumination des vitrines sur l'orientation visuelle des passants

Assistance au parcours : l'entraînement du lumineux. Si la signalétique du Grand Louvre n'opère pas toujours au mieux, elle est relayée par certains dispositifs spatiolumineux qui assurent une véritable assistance au parcours. A cet égard, il est possible de distinguer deux cas exemplaires.

La paroi granulée. Le mur d'enceinte proche du Fossé CharlesV est traité de manière à susciter la curiosité et attirer les visiteurs. La couleur de l'éclairage et l'orientation des sources lumineuses révèlent la texture particulière de ce mur et accentuent le contraste qu'il entretient avec le reste des surfaces lisses et polies (photo 4).

\footnotetext{
4 Dans un essai consacré à la marche en ville, James Hillman (1980) insiste sur l'importance de la dissociation de l'oeil et du pas pour un environnement urbain de qualité : "In the art of the garden, it was considered essential that both the eye and the foot be satisfied: the eye to see, the foot to travel through; the eye to encompass the whole and know it, the foot to remain within it and experience it. It was equally essential in this "aesthetics of dissociation," as Robert Dupree describes it, that the eye and the foot not travel the same path."
} 
Pour citer ce document :

THIBAUD, Jean-Paul. Mouvement et perception des ambiances souterraines. Les annales de la recherche urbaine, juin 1996, $\mathrm{n}^{\circ} 71$, pp. 144-152.

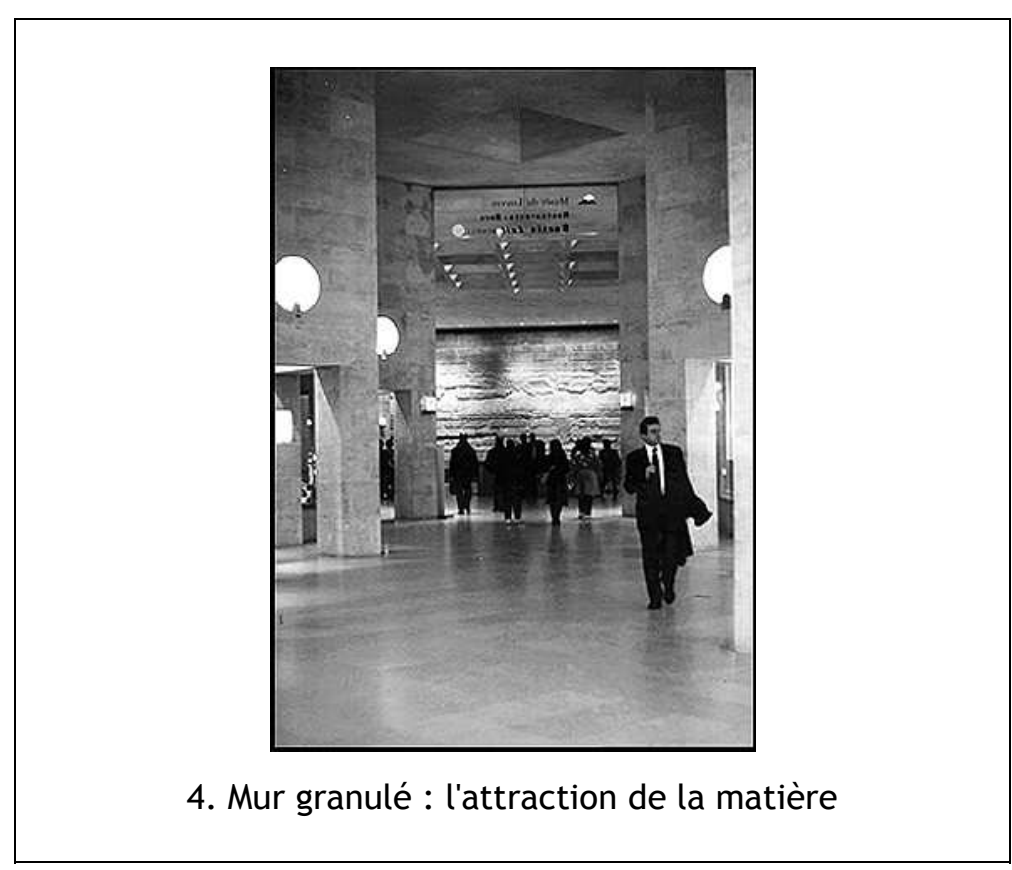

De la Place de la pyramide inversée, les visiteurs ne manquent pas de remarquer ce mur ancien situé au fond de l'Allée du Carrousel : "au fond, il y a un mur qui vraisemblablement est un mur très ancien, donc c'est assez bizarre, c'est étrange, parce que ça fait un... on peut aller voir", "j'ai tout de suite envie de voir, de regarder de près, on dirait une muraille, je ne savais pas qu'il y avait des murailles par là, et surtout sous cette forme", "ça attire l'oeil parce que c'est un mur, on voit bien que c'est un vrai mur". Cette mise en scène du "grain" de la cloison produit un effet de surprise qui suscite inévitablement le commentaire, appelle tout autant l'oeil du passant que son pas et sa main. En favorisant l'émergence et la prégnance d'un événement lumineux, ce dispositif fonctionne comme un embrayeur optomoteur. La vision lointaine permet le surgissement de l'événement mais demande à être relayée par une vision proche et une appréhension tactile qui accomplissent pleinement l'expérience du mur granulés. Cette surface lumineuse mobilise ainsi une vision haptique qui entraîne avec elle l'ensemble du corps.

Les puits de lumière. La pénétration de la lumière naturelle dans l'espace souterrain constitue sans doute un des phénomènes les plus remarquables de ce site. De jour, les visiteurs qui empruntent les galeries menant à la place de la pyramide inversée mentionnent très tôt une grande surface blanche visible même de loin. En particulier, l'obscurité relative de l'Allée du Grand Louvre exacerbe le contraste entre l'éclairage artificiel à proximité et l'éclairage naturel de la pyramide inversée qui

\footnotetext{
5 La perception de la texture d'une surface lumineuse est relative à la distance qui la sépare de l'observateur. Sur l'importance de la texture dans la perception visuelle, se reporter à Roger Ninio (1989).
} 
Pour citer ce document :

THIBAUD, Jean-Paul. Mouvement et perception des ambiances souterraines. Les annales de la recherche urbaine, juin 1996, $\mathrm{n}^{\circ} 71$, pp. 144-152.

s'imprime en fond : "une galerie qui t'amène vers un puits de lumière", "on a le regard fixé par la transparence", "les gens sont attirés par la lumière", "un parcours rythmé, comme ça, un petit guide" (photo 5). 
Pour citer ce document :

THIBAUD, Jean-Paul. Mouvement et perception des ambiances souterraines. Les annales de la recherche urbaine, juin 1996, $\mathrm{n}^{\circ} 71$, pp. 144-152.

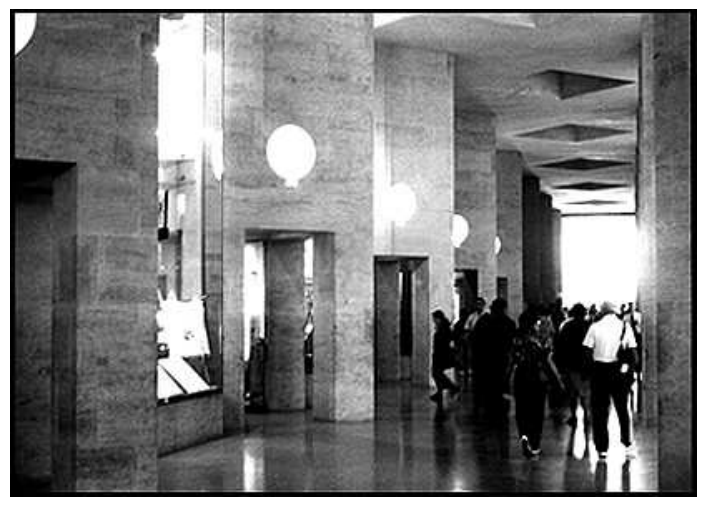

5. Puits de lumière : le guidage de la lumière

Dans ce cas, ce n'est plus la texture de la surface lumineuse qui attire l'oeil du passant, c'est l'éclat de la lumière elle-même. Le regard tend à se fixer et à se focaliser sur cette percée de lumière, comme si l'on ne pouvait pas se dérober à son emprise. Ainsi, les magasins du musée qui bordent cette galerie sont peu évoqués. Celle-ci est essentiellement vécue comme un espace intermédiaire, un lieu de transition dont la fonction première est de donner accès à la place. Un fil imaginaire guide et relie le corps du visiteur à la surface lumineuse lointaine. Anticipant l'arrivée sur la place, le corps du marcheur est tout entier projeté de l'avant, désireux de découvrir ce que l'oeil et la parole ne font qu'annoncer ${ }^{6}$.

Le passage de l'éclairage artificiel à l'éclairage naturel participe du marquage de l'espace et favorise l'expérience des seuils. Plus précisément, la transition vers un espace éclairé à la lumière du jour est vécue généralement sur le mode de la sortie : "là, on a l'impression de sortir, de déboucher dans un coin vraiment de lumière". Quand cela est effectivement le cas, cette configuration lumineuse aide le passant à s'orienter et à trouver son chemin vers l'extérieur (photo 6). La perception de la lumière naturelle coïncide avec l'existence effective d'une sortie.

\footnotetext{
6 Jean-François Augoyard (1979) parle à cet égard de tendance protensionnelle : "La tendance protensionnelle s'exprime lorsque le cheminement se tend déjà tout entier vers le but, lorsque le mouvement, affairé et parfois fébrile, téléscope la succession topologique des lieux (c'est en particulier le cas des figures centrées sur un lieu fascinant), lorsque enfin aucune demeure véritable n'est mémorable dans l'ensemble de la vie quotidienne et que l'habitant saute sans arrêt d'un lieu à un autre."
} 
Pour citer ce document :

THIBAUD, Jean-Paul. Mouvement et perception des ambiances souterraines. Les annales de la recherche urbaine, juin 1996, $\mathrm{n}^{\circ} 71$, pp. 144-152.

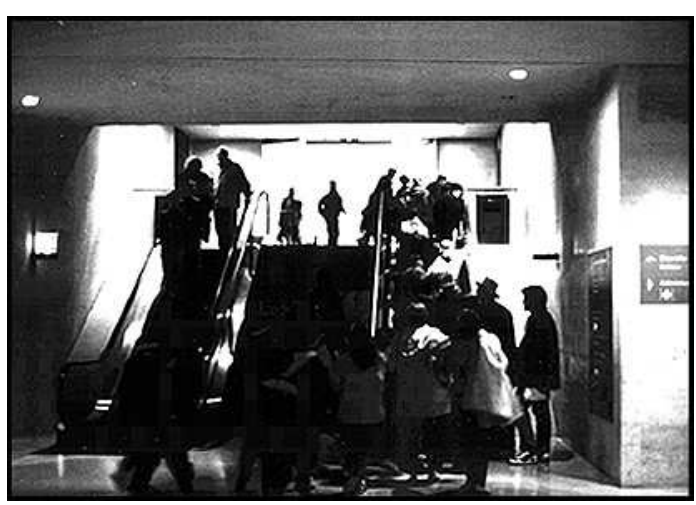

6. Puits de lumière : une sortie vers la rue de Rivoli

Mais il arrive aussi que l'inverse se produise : le puits de lumière instrumente alors l'entrée dans le souterrain (photo 7).

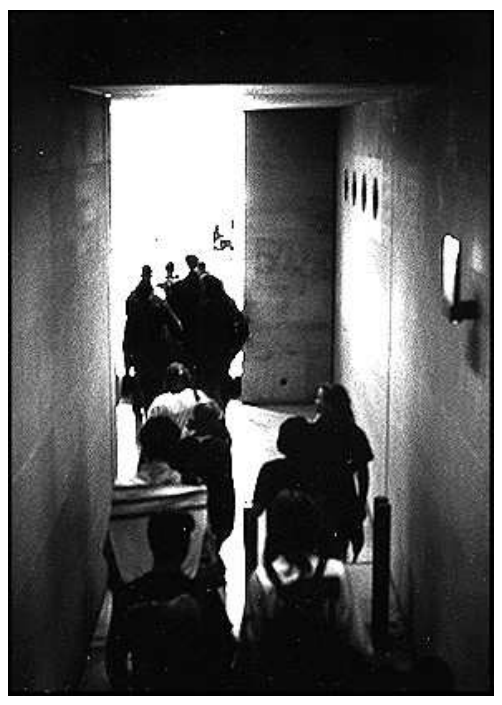

7. Puits de lumière : une entrée dans le Hall Napoléon

Paradoxalement, l'expérience de la descente et de l'immersion dans le sous-sol se combine à la sensation de déboucher sur un espace extérieur. Ce mouvement d'inversion du dedans et du dehors, de superposition simultanée d'un seuil d'entrée et de "sortie" remet en cause l'association de l'enfouissement à l'obscurité. Dans ce cas, le passant ne peut plus s'appuyer sur les indices lumineux de la "profondeur" souterraine pour s'orienter dans l'espace. 
Pour citer ce document :

THIBAUD, Jean-Paul. Mouvement et perception des ambiances souterraines. Les annales de la recherche urbaine, juin 1996, $n^{\circ} 71$, pp. 144-152.

Déstabilisation du pas : la défalllance de l'oeil. La surface lumineuse du sol permet généralement au visiteur d'évaluer les possibilités de déplacement en délivrant des indices sur le degré de solidité et de rigidité du terrain. Toutefois, la vision du passant peut parfois être mise en défaut et produire une déstabilisation du pas, une hésitation temporaire du marcheur.

Le sol miroitant. Le cas du Fossé Charles $\mathrm{V}$ offre un exemple du caractère problématique de ce contrôle opto-moteur. La réflexion de la lumière du plafond sur le sol produit une dilatation verticale de l'espace visuel et créé une ambiguïté quant à la nature du support matériel sur lequel le passant est supposé marcher : "on voit les reflets du plafond, donc ça donne aussi une espèce de dimension, on a l'impression d'être sur... un fleuve, sur une rivière, il y a un reflet permanent ", "ça me fait un peu penser à... une promenade nocturne au bord de la mer" (photo 8). Les hésitations de la parole en recherche du terme adéquat (traduites par les points de suspension) et les expressions employées par les visiteurs ("ça me fait penser à", "on a l'impression") indiquent une incertitude quant à la réalité de ce qui est perçu ; l'association du sol à une matière liquide ("mer", "fleuve") questionne la fermeté du plan horizontal.

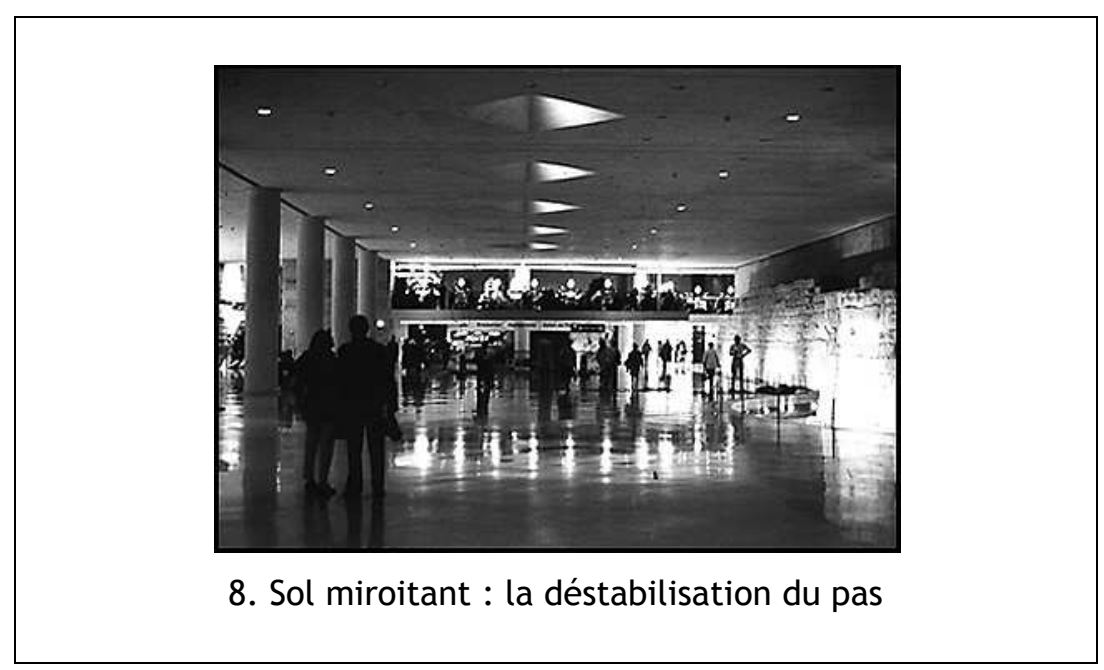

Le compte rendu détaillé d'une passante témoigne de l'interaction entre la défaillance momentanée de l'oeil, le reflet de la lumière sur le sol et l'indécision du pas : "ah tiens, là j'ai eu un moment d'arrêt, je ne sais si j'ai quelque chose avec mes lunettes... en tout cas, c'est comme si c'était de l'eau et comme ça se reflétait... j'ai envie d'aller, de marcher dessus et de voir ; je ne ralentis pas exprès, j'ai envie de voir, il y a comme un îlot de miroirs qui changent, je suppose que la lumière du haut se déplace avec mes mouvements à moi ". L'expérience du sol miroitant se fait sur le mode du jeu, du test et de la vérification : les pauses et mouvements du pas s'effectuent en fonction des transformations observables de la 
Pour citer ce document :

THIBAUD, Jean-Paul. Mouvement et perception des ambiances souterraines. Les annales de la recherche urbaine, juin 1996, $\mathrm{n}^{\circ} 71$, pp. 144-152.

surface lumineuse. La démarche vacille en même temps que la lumière chancelle, l'effet de miroir du sol entraîne le bégaiement des gestes et l'inflexion de la parole.

InVITE à la PAUSE : L'EMPRISE du SPECTACLE. Les configurations lumineuses du Grand Louvre jouent enfin de la verticalité de l'espace en offrant des points de vue remarquables qui invitent à la pause. Selon les cas, le visiteur devient observateur ou spectateur, il prend le temps de regarder et d'apprécier les perspectives qui lui sont proposées.

La fenêtre sur le ciel. L'absence d'horizon de l'espace souterrain accentue le sentiment de confinement. Ce blocage de la portée du regard conduit le passant à rechercher les occasions de vision lointaine. A cet égard, l'échappée visuelle offerte par la "fenêtre" de la pyramide inversée permet de se soustraire au cloisonnement de l'espace. Arrivés place de la pyramide inversée, les passants lèvent immédiatement la tête pour porter leur regard vers le ciel. Sans concertation aucune, ils adoptent tous cette conduite de contemplation du paysage aérien. Parfois même - en particulier avec les enfants - le mouvement de la tête se prolonge par un geste ascensionnel de l'ensemble du corps (photo 9). Orienté vers le haut et débouchant sur l'extérieur, le regard pallie l'impossibilité du corps à sortir du sous-sol.

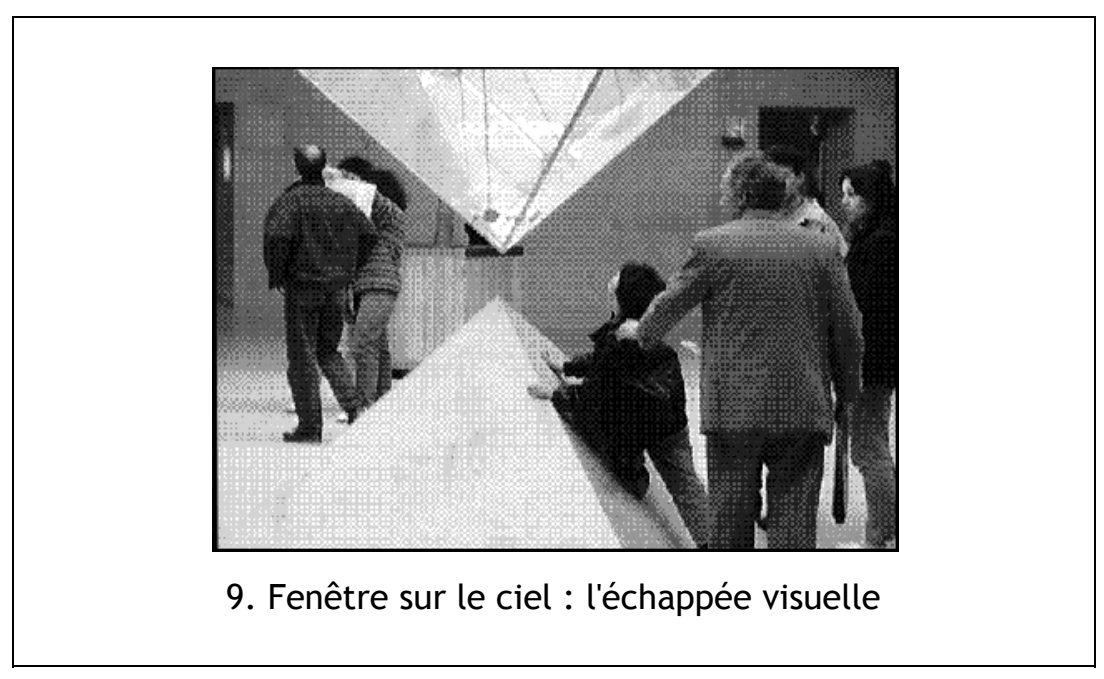

Tout se passe comme si le passant ne pouvait s'empêcher de regarder en haut : "là je suis happée par les nuages", "un plafond avec le ciel", "ça fait comme une fenêtre", "le ciel de la pyramide ce serait comme un tableau, on aperçoit le bleu du ciel avec les nuages". Cet appel du regard vers l'extérieur permet de prendre la mesure du caractère souterrain du site. La vue en contre-plongée qu'offre ce dispositif invite à une vision contemplative qui se traduit par l'abondance des commentaires et se solde souvent par une interruption du pas. En outre, les jeux de lumière que produit la pyramide inversée ne manquent pas d'être décrits avec force détails et invitent le passant à un séjour prolongé. Ici, le temps de la contemplation va de pair avec le repos du corps, le pas du passant s'arrête tout comme l'oeil sur le paysage. 
Pour citer ce document :

THIBAUD, Jean-Paul. Mouvement et perception des ambiances souterraines. Les annales de la recherche urbaine, juin 1996, $n^{\circ} 71$, pp. 144-152.

Les loges du public. La plupart du temps, les visiteurs qui entrent sous la pyramide par la cours Napoléon marquent un temps d'arrêt et mentionnent la présence des passants situés au sous-sol. Les nouveaux arrivants ne semblent pas pouvoir faire autrement que d'observer ce qui se passe en contrebas (photo 10). Ce belvédère d'entrée produit un effet d'attraction tout en rendant le visiteur captif de celui-ci en lui offrant un véritable spectacle : "quand on s'avance on est attiré par un puits, c'est plutôt fait pour qu'on profite du spectacle", "je pense que cette plate-forme est conçue pour qu'on s'y arrête", "là, on surplombe la grande arène", "d'habitude on a une impression de tableau vivant", "on peut voir tout le monde à l'intérieur". Les termes comme "arène", "belvédère" ou "balcon panoramique" rendent compte du point de vue privilégié qu'occupe le nouvel arrivant. En offrant une vision plongeante de l'activité du hall d'accueil et en proposant un sous-sol lumineux uniformément clair qui surexpose les passants situés en contrebas, ce dispositif met en scène la présence du public. Transformé momentanément en spectateur, le nouvel arrivant assiste à une représentation à laquelle lui seul décide de mettre fin.

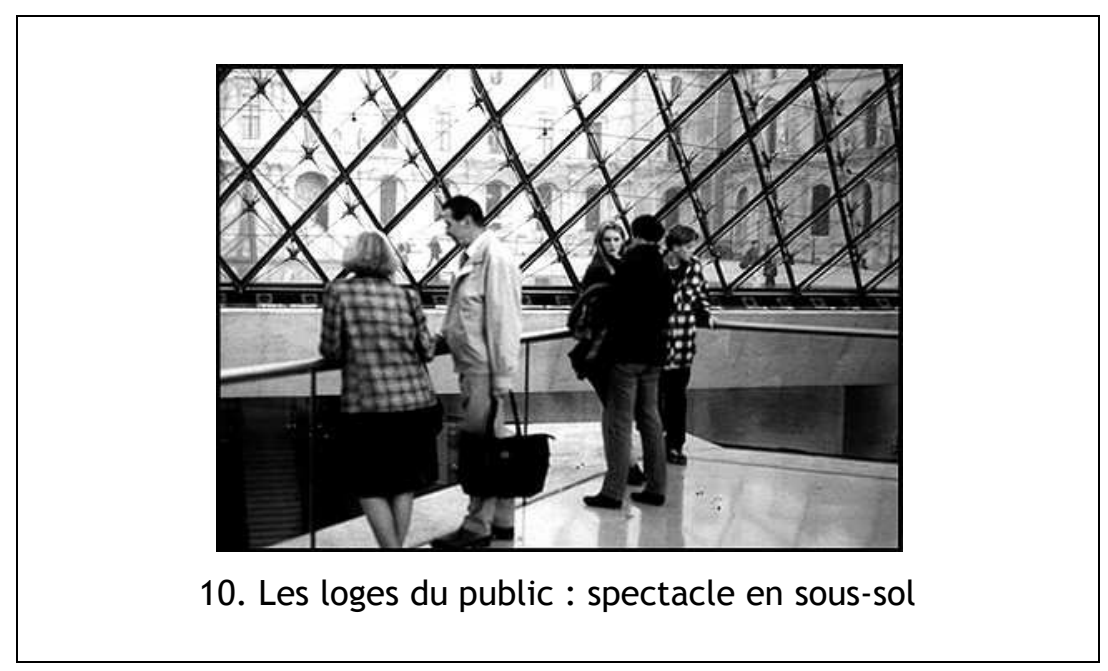

\section{L'incorporation de l'environnement lumineux}

Les dispositifs que nous venons de décrire permettent de préciser le lien qui se tisse entre la mise en lumière du site, la mise en mouvement du corps et la mise en récit de l'expérience. Chacun de ces dispositifs souterrains intervient sur la qualité des surfaces lumineuses, qu'elles soient frontales, latérales, horizontales ou zénithales. C'est à partir de ses qualités de surface - texture, luminosité, réflexion, contraste, etc. - que l'environnement lumineux du site devient véritablement expressif, qu'il offre des prises à l'action et se rend disponible sous forme d'événements. Selon les cas, les surfaces lumineuses expriment et incarnent des 
Pour citer ce document :

THIBAUD, Jean-Paul. Mouvement et perception des ambiances souterraines. Les annales de la recherche urbaine, juin 1996, $\mathrm{n}^{\circ} 71$, pp. 144-152.

possibilités de déplacement, des dispositions de parcours, des invites au séjour. Ce pouvoir expressif des surfaces n'a pas de réalité en soi, indépendamment d'un langage qui les individue, d'une visée intentionnelle qui se projette en elles et d'une performance motrice qui les incorpore?

Pour que l'occurence d'une surface lumineuse devienne événement, soit identifiée et agencée comme telle, il faut qu'une langue s'en saisisse. La "prise de la langue sur les choses" (Gadamer) permet l'expérience du monde en la dotant d'une structure de perspective et d'une temporalité spécifiques. Qualifier une surface de "puits de lumière" ou utiliser la métaphore du fleuve pour relever la prégnance d'un sol réfléchissant, prendre le temps de décrire en détail l'activité d'une place ou n'évoquer que brièvement une galerie souterraine, marquer sa surprise à la vue d'un mur ancien ou faire abstraction d'une partie du cheminement constituent autant d'exemples du pouvoir articulatoire du récit. Le monde à portée de regard émerge et s'agence sur la base d'opérations langagières telles que nommer et réciter. Formulé autrement, la mise en récit de l'environnement lumineux concourt à la structuration temporelle de l'attention.

A cet égard, les qualités lumineuses du site donnent lieu à divers modes d'orientation visuelle ${ }^{8}$ se distribuant au cours du parcours. L'activité perceptive du passant repose sur des gestes attentionnels - focaliser, observer, contempler, examiner, viser, etc. - qui engagent le corps dans sa totalité. Très souvent, le pas du marcheur prend le relai d'un simple mouvement de tête, il accomplit et prolonge ce que le regard ne fait qu'ébaucher. Ce pouvoir moteur des orientations visuelles met en jeu les composantes rythmiques du corps, en oeuvre à la fois dans la perception et dans la mobilité. C'est ainsi que nous parlerons d'effets opto-moteurs pour désigner les phénomènes d'attraction, de guidage, de déstabilisation, d'entraînement ou d'immobilisation que nous avons décrits précédemment.

\section{Bibliographie}

AugoYARD, J.F. Pas à pas. Essai sur le cheminement quotidien en milieu urbain. Le Seuil, Paris, 1979

Barbaras, R. Motricité et phénoménalité chez le dernier Merleau-Ponty. Merleau-Ponty, phénoménologie et expériences. Jérôme Millon, Grenoble, 1992, pp. 27-42

Benjamin, W. Paris, capitale du XIXème siècle. Editions du Cerf, Paris, 1989

\footnotetext{
7 Comme l'a montré Charles Taylor (1979), une expression authentique ne se réduit pas à une simple manifestation, elle met en jeu une incorporation qui la rend possible : "an expression makes something manifest in an embodiment".

8 Sur la notion d'orientation visuelle, se reporter en particulier à l'article de Jeff Coulter et E.D. Parsons (1990).
} 
Pour citer ce document :

THIBAUD, Jean-Paul. Mouvement et perception des ambiances souterraines. Les annales de la recherche urbaine, juin 1996, $n^{\circ} 71$, pp. 144-152.

Coulter, J. and Parsons, E. D. The Praxiology of Perception: Visual Orientations and Practical Action. Inquiry. An Interdisciplinary Journal of Philosophy. Vol. 33, No 3, September 1990, pp. 251-272

GadAMer, H. G. Vérité et Méthode. Le Seuil, Paris, 1976

Gare du Nord : Mode d'emploi. Programme de recherches concertées Plan Urbain - SNCF RATP, $\mathrm{n}^{\circ}$ 96, Paris, 1994

Gibson, J.J. The ecological approach to visual perception. Lawrence Erlbaum Associates, Hillsdale - New Jersey, 1986

Hillman, J. Walking. The City as Dwelling: Walking, Sitting, Shaping. The Center for Civic Leadership, The University of Dallas, Irving, Texas, 1980, pp. 1-7

Merleau-Ponty, M. Le visible et l'invisible. Gallimard, Paris, 1964

Mumford, L. Technics and Civilization. Harcourt, Brace and World, New York, 1934

Ninıo, R. L'empreinte des sens. Odile Jacob/Le Seuil, Paris, 1989

Quéré, L. L'espace public comme forme et comme événement. Prendre place. espace public et culture dramatique. textes réunis par Isaac Joseph, Editions Recherches/Plan Urbain, Paris, 1995, pp. 93-110

Straus, E. Du sens des sens. Jérôme Millon, Grenoble, 1989

TAYLOR, C. Action as expression. Intention and Intentionality. Diamond, C. and Teichman, J. (eds), The Harvester Press, 1979, pp. 73-90

Thibaud, J.P. La méthode des parcours commentés. L'espace urbain en méthodes. sous la direction de Jean-Paul Thibaud et Michèle Grosjean, Parenthèses, 2001 